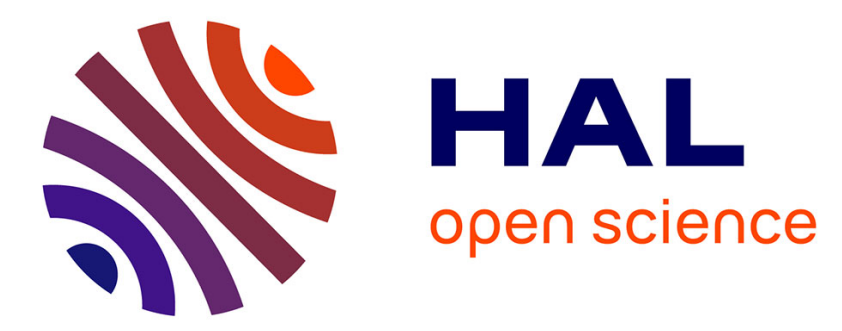

\title{
Bonding a linearly piezoelectric patch on a linearly elastic body
}

Christian Licht, Somsak Orankitjaroen, Patcharakorn Viriyasrisuwattana, Thibaut Weller

\section{- To cite this version:}

Christian Licht, Somsak Orankitjaroen, Patcharakorn Viriyasrisuwattana, Thibaut Weller. Bonding a linearly piezoelectric patch on a linearly elastic body. Comptes Rendus Mécanique, 2014, 342 (4), pp.234-239. 10.1016/j.crme.2014.01.003 . hal-01233081

\section{HAL Id: hal-01233081 \\ https://hal.science/hal-01233081}

Submitted on 24 Nov 2015

HAL is a multi-disciplinary open access archive for the deposit and dissemination of scientific research documents, whether they are published or not. The documents may come from teaching and research institutions in France or abroad, or from public or private research centers.
L'archive ouverte pluridisciplinaire HAL, est destinée au dépôt et à la diffusion de documents scientifiques de niveau recherche, publiés ou non, émanant des établissements d'enseignement et de recherche français ou étrangers, des laboratoires publics ou privés. 


\title{
Bonding a linearly piezoelectric patch on a linearly elastic body
}

\section{Analyse asymptotique d'un patch linéairement piézoélectrique lié à un corps linéairement élastique}

\author{
Christian Licht ${ }^{a, b, c, *}$, Somsak Orankitjaroen ${ }^{b, c}$, \\ Patcharakorn Viriyasrisuwattana ${ }^{\mathrm{b}, \mathrm{c}}$, Thibaut Weller ${ }^{\mathrm{a}, *}$ \\ a LMGC, UMR-CNRS 5508, Université Montpellier-2, case courier 048, place Eugène-Bataillon, 34095 Montpellier cedex 5, France \\ b Department of Mathematics, Faculty of Science, Mahidol University, Bangkok 10400, Thailand \\ c Centre of Excellence in Mathematics, CHE, Bangkok 10400, Thailand
}

\section{A B S T R A C T}

A rigorous study of the asymptotic behavior of the system constituted by a very thin linearly piezoelectric plate bonded on a linearly elastic body supplies various models for an elastic body monitored by a piezoelectric patch.

\section{R É S U M É}

Une étude rigoureuse du comportement asymptotique du système constitué par une plaque linéairement piézoélectrique collée sur un corps linéairement élastique fournit divers modèles de contrôle de structures élastiques par des patches piézoélectriques.

\section{Introduction}

Many studies dealing with the mathematical modeling of piezoelectric devices were devoted to the behavior of the sole patches and provided various asymptotic models for thin linearly piezoelectric plates (see [1] and the references therein). However, the essential technological interest of piezoelectric patches being the monitoring of a deformable body they are bonded to, here we intend to propose various asymptotic models for the behavior of the body through the study of the system constituted by a very thin linearly piezoelectric flat patch perfectly bonded to a linearly elastic three-dimensional body. A reference configuration for the body is an open set $\Omega$ laying in $\left\{x_{3}<0\right\}$ whose part of its Lipschitz-continuous boundary $\partial \Omega$ is a non-empty domain $S$ in $\left\{x_{3}=0\right\}$ and such that $S \times(-L, 0)$ is included in $\Omega$ for some positive real number $L$, while the patch occupies $B^{\varepsilon}:=S \times(0, \varepsilon), \varepsilon$ being a small real number; let $\mathcal{O}^{\varepsilon}:=\Omega \cup S \cup B^{\varepsilon}$. The body is clamped on a part $\Gamma_{0}$ 
of $\partial \Omega \backslash S$ with a positive two-dimensional Hausdorff measure $\mathcal{H}_{2}\left(\Gamma_{0}\right)$, and subjected to body forces and surface forces on $\Gamma_{1}:=\partial \Omega \backslash\left(S \cup \Gamma_{0}\right)$ of densities $f$ and $F$. Moreover, for all $\delta$ in $\mathbb{R}$, let $S^{\delta}$ denote $S+\delta e_{3},\left\{e_{1}, e_{2}, e_{3}\right\}$ being a basis of the Euclidean physical space assimilated to $\mathbb{R}^{3}$, surface forces of density $G$ acts on $S^{\varepsilon}$ whilst the patch is free of mechanical loading and electric charges in $B^{\varepsilon}$ and on its lateral boundary $\partial S \times(0, \varepsilon)$. If $u^{\varepsilon}, e\left(u^{\varepsilon}\right), \sigma^{\varepsilon}$ denote the fields of displacement, strain and stress in $\mathcal{O}^{\varepsilon}$ and $\varphi^{\varepsilon}, D^{\varepsilon}$ stand for the electric potential and the electric displacement, part of the equations describing the electromechanical equilibrium read as:

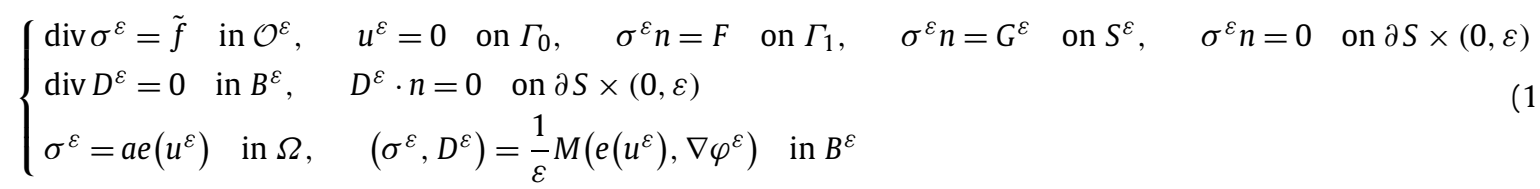

$\tilde{f}$ is the extension of $f$ to $B^{\varepsilon}$ by $0, n$ is the unit outward normal and $a$ denotes the elasticity tensor which satisfies:

$$
a \in L^{\infty}\left(\Omega ; \operatorname{Lin}\left(\mathbb{S}^{3}\right)\right), \quad \exists c ; c|e|^{2} \leqslant a(x) e \cdot e, \quad \forall e \in \mathbb{S}^{3} \text {, a.e. } x \in \Omega
$$

where Lin $\left(\mathbb{S}^{N}\right)$ is the space of linear operators on the space $\mathbb{S}^{N}$ of $N \times N$ symmetric matrices whose inner product and norm are noted and $|\cdot|$ as in $\mathbb{R}^{3}$. If $\mathbb{H}:=\mathbb{S}^{3} \times \mathbb{R}^{3}$ is equipped with an inner product and a norm also denoted as previously, then $M$ is an element of $L^{\infty}(S \times \mathbb{R} ; \operatorname{Lin}(\mathbb{H}))$ independent of $x_{3}$ satisfying:

$$
M=\left[\begin{array}{cc}
\alpha & -\beta \\
\beta^{T} & \gamma
\end{array}\right], \quad \exists \kappa>0 ; \quad \kappa|e|^{2} \leqslant \alpha(x) e \cdot e, \quad \kappa|g|^{2} \leqslant \gamma(x) g \cdot g, \quad \forall(e, g) \in \mathbb{H}, \text { a.e. } x \in S \times \mathbb{R}
$$

The models will be distinguished according to the additional necessary boundary conditions on $S^{\varepsilon}$ and $S$, characterized by an index $p$ in $\{1,2\}^{2}$. Case $p_{1}=1$ corresponds to a condition for the electric displacement on $S^{\varepsilon}$ :

$$
D^{\varepsilon} \cdot n=q^{\varepsilon} \quad \text { on } S^{\varepsilon}
$$

$q^{\varepsilon}$ being a density of electrical charges, while $p_{1}=2$ corresponds to a condition of given electrical potential:

$$
\varphi^{\varepsilon}=\varphi_{0}^{\varepsilon} \quad \text { on } S^{\varepsilon}
$$

roughly speaking, $p_{1}=1$ deals with patches used as sensors, whereas $p_{1}=2$ concerns actuators (see [1,2]). Index $p_{2}$ accounts for the status of the interface between the patch and the body: $p_{2}=1$ corresponds to an electrically impermeable interface, $p_{2}=2$ corresponds to a grounded interface:

$$
\begin{aligned}
& D^{\varepsilon} \cdot n=0 \text { on } S \\
& \varphi^{\varepsilon}=0 \text { on } S
\end{aligned}
$$

It will be convenient to use the following notations:

$$
\left\{\begin{array}{l}
\hat{k}:=(\hat{e}, \hat{g}), \quad \hat{e}:=\left(e_{\alpha \beta}\right)_{\alpha, \beta \in\{1,2\}}, \quad \hat{g}:=\left(g_{1}, g_{2}\right), \quad \forall k=(e, g) \in \mathbb{H} \\
\tilde{e} \in \mathbb{S}^{3} ; \quad \tilde{e}_{\alpha \beta}=e_{\alpha \beta}, \quad 1 \leqslant \alpha, \beta \leqslant 2, \quad \tilde{e}_{i 3}=0, \quad 1 \leqslant i \leqslant 3, \forall e \in \mathbb{S}^{2} \\
k(r)=k(v, \psi):=(e(v), \nabla \psi), \quad \forall r=(v, \psi) \in H^{1}\left(B^{\varepsilon} ; \mathbb{R}^{3}\right) \times H^{1}\left(B^{\varepsilon}\right) \\
e(v) \in \mathcal{D}^{\prime}\left(S ; \mathbb{S}^{2}\right) ; \quad(e(v))_{\alpha \beta}=\frac{1}{2}\left(\partial_{\alpha} v_{\beta}+\partial_{\beta} v_{\alpha}\right), \quad \forall v \in \mathcal{D}^{\prime}\left(S ; \mathbb{R}^{2}\right)
\end{array}\right.
$$

where the same symbol $e(\cdot)$ stands for the symmetrized gradient in the sense of distributions of $\mathcal{D}^{\prime}\left(\mathcal{O} ; \mathbb{R}^{3}\right), \mathcal{O} \in$ $\left\{\Omega, B^{\varepsilon}, \mathcal{O}^{\varepsilon}\right\}$, or $\mathcal{D}^{\prime}\left(S ; \mathbb{R}^{2}\right)$. Moreover we introduce some spaces, linear and bilinear forms in order to supply a variational formulation of (1)-(5). An electromechanical state will be an element $r=(v, \psi)$ of

$$
V_{p}:=H_{\Gamma_{0}}^{1}\left(\mathcal{O}^{\varepsilon} ; \mathbb{R}^{3}\right) \times \Phi_{p}, \quad \Phi_{(1,1)}=H^{1}\left(B^{\varepsilon}\right), \quad \Phi_{(1,2)}=H_{S}^{1}\left(B^{\varepsilon}\right), \quad \Phi_{(2,1)}=H_{S^{\varepsilon}}^{1}\left(B^{\varepsilon}\right), \quad \Phi_{(2,2)}=H_{S \cup S^{\varepsilon}}^{1}\left(B^{\varepsilon}\right)
$$

where, for any domain $\mathcal{O}$ of $\mathbb{R}^{3}, H_{\Gamma}^{1}\left(\mathcal{O} ; \mathbb{R}^{3}\right)$ and $H_{\Gamma}^{1}(\mathcal{O})$ respectively denote the subspaces of $H^{1}\left(\mathcal{O} ; \mathbb{R}^{3}\right)$ and $H^{1}(\mathcal{O})$ of all elements with vanishing traces on a part $\Gamma$ of $\partial \mathcal{O}$. One makes the following assumptions on the data:

$$
\left\{\begin{array}{l}
\varphi_{0} \text { denotes the restriction to } S \text { of an element of } H^{1 / 2}\left(\left\{x_{3}=0\right\}\right) \text { still denoted by } \varphi_{0} \\
(f, F, G, q) \in L^{2}\left(\Omega ; \mathbb{R}^{3}\right) \times L^{2}\left(\Gamma_{1} ; \mathbb{R}^{3}\right) \times L^{2}\left(S ; \mathbb{R}^{3}\right) \times L^{2}(S), \quad \int_{S} q \mathrm{~d} \hat{x}=0 \\
G^{\varepsilon}\left(x+\varepsilon e_{3}\right)=G(x), \quad q^{\varepsilon}\left(x+\varepsilon e_{3}\right)=q(x), \quad \varphi_{0}^{\varepsilon}\left(x+\varepsilon e_{3}\right)=\varepsilon \varphi_{0}(x), \quad \text { a.e. } x \in S
\end{array}\right.
$$

It is well known that for all $\varphi_{0}$ in $H^{1 / 2}\left(\left\{x_{3}=0\right\}\right)$, there exists an element of $H^{1}(S \times(-L, 0))$ when $p_{2}=1, H_{S^{-L}}^{1}(S \times(-L, 0))$ when $p_{2}=2$, still denoted by $\varphi_{0}$ whose trace on $S$ is $\varphi_{0}$. Hence the element $\varphi_{o, p}^{\varepsilon}$ of $\Phi_{p}$ defined by $\varphi_{0, p}^{\varepsilon}(x)=\varepsilon \varphi_{0}\left(\hat{x}\right.$, $\left(x_{3}-\right.$ $\varepsilon) L / \varepsilon$ ) satisfies: 


$$
\varphi_{o, p}^{\varepsilon}:=\varphi_{0}^{\varepsilon} \quad \text { on } S^{\varepsilon}, \quad \frac{1}{\varepsilon} \int_{B^{\varepsilon}}\left|\nabla \varphi_{o, p}^{\varepsilon}\right|^{2} \mathrm{~d} x \leqslant C
$$

Let $\mathcal{M}_{p}$ and $\mathcal{L}_{p}$ be defined by:

$$
\begin{aligned}
& \mathcal{M}_{p}(s, r):=\int_{\Omega} a e(u) \cdot e(v) \mathrm{d} x+\frac{1}{\varepsilon} \int_{B^{\varepsilon}} M k(s) \cdot k(r) \mathrm{d} x, \quad \forall s=(u, \varphi), \forall r=(v, \psi) \in V_{p} \\
& \mathcal{L}_{\left(1, p_{2}\right)}(r)=\int_{\Omega} f \cdot v \mathrm{~d} x+\int_{\Gamma_{1}} F \cdot v \mathrm{~d} H_{2}+\int_{S^{\varepsilon}} G^{\varepsilon} \cdot v \mathrm{~d} \hat{x}+\int_{S^{\varepsilon}} q^{\varepsilon} \cdot \psi \mathrm{d} \hat{x}, \quad \forall r=(v, \psi) \in V_{\left(1, p_{2}\right)} \\
& \mathcal{L}_{\left(2, p_{2}\right)}(r)=\int_{\Omega} f \cdot v \mathrm{~d} x+\int_{\Gamma_{1}} F \cdot v \mathrm{~d} H_{2}+\int_{S^{\varepsilon}} G^{\varepsilon} \cdot v \mathrm{~d} \hat{x}-\frac{1}{\varepsilon} \int_{B^{\varepsilon}} M k\left(0, \varphi_{o, p}^{\varepsilon}\right) \cdot k(r) \mathrm{d} x, \quad \forall r=(v, \psi) \in V_{\left(2, p_{2}\right)}
\end{aligned}
$$

It is straightforward to check that $\mathcal{M}_{p}$ is continuous and coercive on $V_{p}$ (actually on $H_{\Gamma_{0}}^{1}\left(\Omega ; \mathbb{R}^{3}\right) \times\left(\Phi_{p} / \mathbb{R}\right)$ when $p=(1,1)$ ), $\mathcal{L}_{p}$ is continuous on $V_{p}$, and seeking an equilibrium state leads to the problem

$$
\left(P_{p}^{\varepsilon}\right): \quad \text { Find } \tilde{s}_{p}^{\varepsilon}=\left(u_{p}^{\varepsilon}, \widetilde{\varphi}_{p}^{\varepsilon}\right) \text { in } V_{p} \text { such that } \mathcal{M}_{p}\left(\tilde{s}_{p}^{\varepsilon}, r\right)=\mathcal{L}_{p}(r), \quad \forall r \in V_{p}
$$

which by the Lax-Milgram lemma has a unique solution (if one adds the condition $\left\langle\widetilde{\varphi}_{p}^{\varepsilon}\right\rangle_{\varepsilon}:=\frac{1}{\left|S^{\varepsilon}\right|} \int_{S^{\varepsilon}} \widetilde{\varphi}_{p}^{\varepsilon} \mathrm{d} \hat{x}=0$ when $p=$ $(1,1))$. Then an equilibrium state is $s_{p}^{\varepsilon}=\tilde{s}_{p}^{\varepsilon}+\left(0, \varphi_{o, p}^{\varepsilon}\right)$.

\section{The asymptotic models}

We will propose our four models by studying the asymptotic behavior of $s_{p}^{\varepsilon}$, when $\varepsilon$ goes to zero, in three steps:

Step 1 (a priori estimates): By taking $r=\tilde{s}_{p}^{\varepsilon}$ in the formulation of $\left(P_{p}^{\varepsilon}\right)$ and by due account of (2), (3), (8), (9) and of the following Lemma 2.1, we have:

$$
\exists C>0 ; \quad \int_{\Omega}\left|e\left(u_{p}^{\varepsilon}\right)\right|^{2} \mathrm{~d} x+\frac{1}{\varepsilon} \int_{B^{\varepsilon}}\left(\left|e\left(u_{p}^{\varepsilon}\right)\right|^{2}+\left|\nabla \varphi_{p}^{\varepsilon}\right|^{2}\right) \mathrm{d} x \leqslant C
$$

Lemma 2.1. There hold:

(i) $|v|_{L^{2}\left(S^{\varepsilon} ; \mathbb{R}^{3}\right)}^{2} \leqslant C\left(|e(v)|_{L^{2}\left(\Omega ; \mathbb{S}^{3}\right)}^{2}+|e(v)|_{L^{2}\left(B^{\varepsilon}, \mathbb{S}^{3}\right)}^{2}\right), \forall v \in H_{\Gamma_{0}}^{1}\left(\mathcal{O}^{\varepsilon} ; \mathbb{R}^{3}\right)$,

(ii) $\int_{S^{\varepsilon}}\left|\psi-\langle\psi\rangle_{\varepsilon}\right|^{2} \mathrm{~d} \hat{x} \leqslant \frac{C}{\varepsilon} \int_{B^{\varepsilon}}|\nabla \psi|^{2} \mathrm{~d} x, \forall \psi \in H^{1}\left(B^{\varepsilon}\right)$,

(iii) $\int_{B^{\varepsilon}} \psi^{2} \mathrm{~d} x \leqslant c\left[\varepsilon \int_{\Gamma} \psi^{2} \mathrm{~d} \hat{x}+\varepsilon^{2} \int_{B^{\varepsilon}}|\nabla \psi|^{2} \mathrm{~d} x\right], \forall \psi \in H^{1}\left(B^{\varepsilon}\right), \forall \Gamma \in\left\{S, S^{\varepsilon}\right\}$.

Proof. Points (ii)-(iii) are standard due to the cylindrical geometry of $B^{\varepsilon}$. To prove (i), it suffices to introduce a $C^{\infty}([-L, L / 3])$ cut-off function $\eta$ such that:

$$
\eta=0 \quad \text { on }\left[-L,-\frac{2 L}{3}\right], \quad \eta=1 \quad \text { on }\left[-\frac{L}{3}, \frac{L}{3}\right], \quad 0 \leqslant \eta \leqslant 1, \quad 0 \leqslant \frac{\mathrm{d} \eta}{\mathrm{d} x_{3}} \leqslant \frac{4}{L} \quad \text { on }\left[-\frac{2 L}{3},-\frac{L}{3}\right]
$$

and to apply the Korn inequality in $H_{S^{-L+\varepsilon}}^{1}\left(S \times(-L+\varepsilon, \varepsilon) ; \mathbb{R}^{3}\right)$ to $\eta v$ by noticing that the constant in Korn's inequality does not depend on $\varepsilon$.

Therefore the Cauchy-Schwarz inequality and (12) make it possible to define the following element of $L^{2}(S ; \mathbb{H})$ :

$$
k\left(\varepsilon, s_{p}^{\varepsilon}\right)=\left(e\left(\varepsilon, u_{p}^{\varepsilon}\right), g\left(\varepsilon, \varphi_{p}^{\varepsilon}\right)\right):=\frac{1}{\varepsilon} \int_{0}^{\varepsilon}\left(e\left(u_{p}^{\varepsilon}\right), \nabla \varphi_{p}^{\varepsilon}\right)\left(\cdot, x_{3}\right) \mathrm{d} x_{3}
$$

which satisfies:

$$
\left|k\left(\varepsilon, s_{p}^{\varepsilon}\right)\right|_{L^{2}(S ; \mathbb{H})} \leqslant C
$$

thus there exists a nonrelabeled subsequence such that $k\left(\varepsilon, s_{p}^{\varepsilon}\right)$ converges weakly in $L^{2}(S ; \mathbb{H})$ toward some $\bar{k}_{p}$. The interest of introducing $k\left(\varepsilon, s_{p}^{\varepsilon}\right)$ is that 


$$
\left.\widehat{k\left(\varepsilon, s_{p}^{\varepsilon}\right.}\right)=\left(e\left(\widehat{U_{p}^{\varepsilon}}\right), \nabla \phi_{p}^{\varepsilon}\right), \quad\left(U_{p}^{\varepsilon}, \phi_{p}^{\varepsilon}\right):=\frac{1}{\varepsilon} \int_{0}^{\varepsilon} s_{p}^{\varepsilon}\left(\cdot, x_{3}\right) \mathrm{d} x_{3}
$$

which will enable us to identify $\bar{k}_{p}$.

\section{Step 2 (Convergence of $\left(s_{p}^{\varepsilon}\right)$ ):}

Proposition 2.1. Let $\bar{V}:=\left\{v \in H_{\Gamma_{0}}^{1}\left(\Omega ; \mathbb{R}^{3}\right) ; \hat{v} \in H^{1}\left(S ; \mathbb{R}^{2}\right)\right\}$, then there exists $\left(\bar{u}_{p}, \bar{\phi}_{p}\right)$ in $\bar{V} \times H^{1}(S)$ such that, when $\varepsilon$ goes to zero,

(i) the restriction to $\Omega$ of $u_{p}^{\varepsilon}$ converges weakly in $H_{\Gamma_{0}}^{1}\left(\Omega ; \mathbb{R}^{3}\right)$ toward $\bar{u}_{p}$;

(ii) $\left(\widehat{U}_{p}^{\varepsilon}, \phi_{p}^{\varepsilon}\right)$ converges weakly in $H^{1}\left(S ; \mathbb{R}^{2} \times \mathbb{R}\right)$, and consequently strongly in $L^{2}\left(S ; \mathbb{R}^{2} \times \mathbb{R}\right)$ toward $\left(\widehat{\bar{u}_{p}}, \bar{\phi}_{p}\right), \widehat{\bar{k}}_{p}=\left(e\left(\widehat{\bar{u}_{p}}\right), \nabla \bar{\phi}_{p}\right)$, and $\bar{\phi}_{p}=0$ when $p \neq(1,1)$.

Proof. The estimate (12) yields that a nonrelabeled subsequence $\left(u_{p}^{\varepsilon}\right)$ has restriction to $\Omega$ which weakly converges in $H_{\Gamma_{0}}^{1}\left(\Omega ; \mathbb{R}^{3}\right)$ and whose trace on $S$ strongly converges in $L^{2}\left(S ; \mathbb{R}^{3}\right)$. Hence, regarding $U_{p}^{\varepsilon}$, it remains to show

$$
\lim _{\varepsilon \rightarrow 0} I_{\varepsilon}=0, \quad I_{\varepsilon}:=\int_{S} \frac{1}{\varepsilon^{2}}\left(\int_{0}^{\varepsilon}\left(\int_{0}^{x_{3}}\left|\partial_{3} u_{p}^{\varepsilon}(\hat{x}, t)\right| \mathrm{d} t\right) \mathrm{d} x_{3}\right)^{2} \mathrm{~d} \hat{x}
$$

The repeated use of the Cauchy-Schwarz inequality leads to $I_{\varepsilon} \leqslant \varepsilon \int_{B^{\varepsilon}}\left|\nabla u_{p}^{\varepsilon}\right|^{2} \mathrm{~d} x$, and (12), (13) yield:

$$
\begin{aligned}
\varepsilon \int_{B^{\varepsilon}}\left|\nabla u_{p}^{\varepsilon}\right|^{2} \mathrm{~d} x & =\varepsilon \int_{B^{\varepsilon}}\left|\nabla\left(\eta u_{p}^{\varepsilon}\right)\right|^{2} \mathrm{~d} x \leqslant \varepsilon \int_{\mathcal{O}^{\varepsilon}}\left|\nabla\left(\eta u_{p}^{\varepsilon}\right)\right|^{2} \mathrm{~d} x \\
& \leqslant C \varepsilon \int_{S \times(-L+\varepsilon, \varepsilon)}\left|e\left(\eta u_{p}^{\varepsilon}\right)\right|^{2} \mathrm{~d} x \leqslant C \varepsilon\left(\left|e\left(u_{p}^{\varepsilon}\right)\right|_{L^{2}\left(\Omega ; \mathbb{S}^{3}\right)}^{2}+\left|e\left(u_{p}^{\varepsilon}\right)\right|_{L^{2}\left(B^{\varepsilon} ; \mathbb{S}^{3}\right)}^{2}\right) \leqslant C \varepsilon
\end{aligned}
$$

Moreover (16) implies that $e\left(U_{p}^{\varepsilon}\right)$ converges in $\mathcal{D}^{\prime}\left(S ; \mathbb{S}^{2}\right)$ toward both $e\left(\hat{u}_{p}\right)$ and $\widehat{\bar{e}}$, thus $\bar{u}_{p}$ belongs to $\bar{V}$ and $\widehat{\bar{e}}=e\left(\widehat{\bar{u}}_{p}\right)$ ! Lastly, as $\int_{S}\left|\phi_{p}^{\varepsilon}\right|^{2} \mathrm{~d} x \leqslant \frac{1}{\varepsilon} \int_{B^{\varepsilon}}\left|\varphi_{p}^{\varepsilon}\right|^{2} \mathrm{~d} x$, (12), Lemma 2.1(iii) and (ii) with $\left\langle\varphi_{(1,1)}^{\varepsilon}\right\rangle_{\varepsilon}=0$ yield that $\phi_{p}^{\varepsilon}$ is bounded in $L^{2}(S)$ and converges strongly toward 0 when $p \neq(1,1)$. In the next step, we will show that $\left(\bar{U}_{p}, \bar{g}_{p}\right)$ is necessarily the unique solution to a variational problem so that the whole sequences do converge.

Step 3 (Identification of $\left(\overline{\boldsymbol{u}}_{\boldsymbol{p}}, \overline{\boldsymbol{\phi}}_{\boldsymbol{p}}\right)$ ): First, the decomposition

$$
\begin{cases}\mathbb{H}=\mathbb{H}_{p}^{1} \oplus \mathbb{H}_{p}^{2} \oplus \mathbb{H}_{p}^{3} \\ p_{1}=1: \quad \mathbb{H}_{p}^{1}:=\left\{h=(e, g) \in \mathbb{H} ; e_{i 3}=0, g_{3}=0\right\}, \quad \mathbb{H}_{p}^{2}:=\{h=(e, g) \in \mathbb{H} ; \hat{e}=0, \hat{g}=0\}, \quad \mathbb{H}_{p}^{3}:=\{0\} \\ p_{1}=2: \quad \mathbb{H}_{p}^{1}=\left\{h=(e, g) \in \mathbb{H} ; e_{i 3}=0, \hat{g}=0\right\}, \quad \mathbb{H}_{p}^{2}=\{(e, g) \in \mathbb{H} ; \hat{e}=0, g=0\}, \\ \quad \mathbb{H}_{p}^{3}=\left\{(e, g) \in \mathbb{H} ; \quad e=0, g_{3}=0\right\}\end{cases}
$$

induces a decomposition of $M$ in linear operators $M_{p}^{i j}$ mapping $\mathbb{H}_{p}^{i}$ into $\mathbb{H}_{p}^{j}$. The key point in the identification of $\left(\bar{u}_{p}, \bar{\phi}_{p}\right)$ is to establish:

$$
\left(M \bar{k}_{p}\right)^{2}=0
$$

where $h^{i}$ denotes the projection on $\mathbb{H}_{p}^{i}$ of any $h$ of $\mathbb{H}$. As (14) and (12) imply:

$$
\left\{\begin{array}{l}
\frac{1}{\varepsilon} \int_{B^{\varepsilon}} M\left(e\left(u_{p}^{\varepsilon}\right), \nabla \varphi_{p}^{\varepsilon}\right) \cdot \theta \mathrm{d} x=\int_{S} M\left(e\left(\varepsilon, u_{p}^{\varepsilon}\right), g\left(\varepsilon, \varphi_{p}^{\varepsilon}\right)\right) \cdot \theta \mathrm{d} \hat{x} \\
\lim _{\varepsilon \rightarrow 0} \frac{1}{\varepsilon} \int_{B^{\varepsilon}} M\left(e\left(u_{p}^{\varepsilon}\right), \nabla \varphi_{p}^{\varepsilon}\right)(x) \cdot x_{3} \theta(\hat{x}) \mathrm{d} x=0, \quad \forall \theta \in L^{2}(S ; \mathbb{H})
\end{array}\right.
$$

equality (19) is a mere consequence of choosing the following test functions in the formulation of $\left(P_{p}^{\varepsilon}\right)$ : 


$$
\left\{\begin{array}{l}
{ }^{I} r=\left({ }^{I} v, 0\right), I=1,2 ; \quad{ }^{I} v=0 \text { in } \Omega, \quad{ }^{1} v=\left(0, x_{3} \theta\right), \quad{ }^{2} v=\left(x_{3} \theta, 0\right), \quad \theta \in C_{0}^{\infty}\left(S ; \mathbb{R}^{I}\right), \text { in } B^{\varepsilon} \\
r=(0, \psi), \quad \psi(x)=x_{3} \theta(\hat{x}), \quad \theta \in C_{0}^{\infty}(S)
\end{array}\right.
$$

Hence, as (18) and point (ii) in Proposition 2.1 yield $\left(\bar{k}_{p}\right)^{3}=0$, we deduce:

$$
\left(M \bar{k}_{p}\right)^{1}=\widetilde{M}_{p}\left(\bar{k}_{p}\right)^{1} ; \quad \widetilde{M}_{p}:=M_{p}^{11}-M_{p}^{12}\left(M_{p}^{22}\right)^{-1} M_{p}^{21}
$$

Next, for all $(v, \psi)$ in $V_{K L}\left(B^{\varepsilon}\right) \times \Psi_{p}$, defined by:

$$
\begin{aligned}
& V_{K L}\left(B^{\varepsilon}\right)=\left\{v \in H^{1}\left(B^{\varepsilon} ; \mathbb{R}^{3}\right) ; \exists\left(v^{M}, v^{F}\right) \in H^{1}\left(S ; \mathbb{R}^{2}\right) \times H^{2}(S) ; \hat{v}(x)=v^{M}(\hat{x})-x_{3} \nabla v^{F}(\hat{x}), v_{3}(x)=v^{F}(\hat{x}),\right. \\
& \left.\quad \text { a.e. } x \in B^{\varepsilon}\right\} \\
& \Psi_{p}=\left\{\psi ; \psi(x)=\left(x_{3}-\varepsilon\right)^{p_{1}-1} \theta(\hat{x}), \theta \in H^{2-p_{1}}(S)\right\} \quad \text { if } p_{2}=1, \quad \Psi_{p}=\{0\} \quad \text { if } p_{2}=2
\end{aligned}
$$

the couple $(\widetilde{e(\hat{v})}, \nabla \psi)$ belongs to $\mathbb{H}_{p}^{1}$ almost everywhere in $S$, then (20) and (22) give:

$$
\left.\lim _{\varepsilon \rightarrow 0} \frac{1}{\varepsilon} \int_{B^{\varepsilon}} M\left(e\left(u_{p}^{\varepsilon}\right), \nabla \varphi_{p}^{\varepsilon}\right) \cdot(e(v), \nabla \psi) \mathrm{d} x=\int_{S} M \bar{k}_{p} \cdot(\widetilde{e(\hat{v})}, \nabla \psi) \mathrm{d} \hat{x}=\int_{S} \widetilde{M}_{p}\left(\bar{k}_{p}\right)^{1} \cdot(e \widetilde{\hat{v}}), \nabla \psi\right) \mathrm{d} \hat{x}
$$

while, obviously, $\lim _{\varepsilon \rightarrow 0} \int_{S^{\varepsilon}} G^{\varepsilon} \cdot v \mathrm{~d} \hat{x}+\int_{S^{\varepsilon}} q^{\varepsilon} \cdot \psi \mathrm{d} \hat{x}=\int_{S} G \cdot v \mathrm{~d} \hat{x}+\int_{S} q \cdot \psi \mathrm{d} \hat{x}$.

Lastly the very definitions of $g\left(\varepsilon, \varphi_{p}^{\varepsilon}\right)$ and $\widetilde{M}_{p}$ implying $\left(\bar{g}_{(2,2)}\right)_{3}=\varphi_{0}$ and $\widetilde{M} k \cdot k \geqslant 2 \kappa|k|^{2}$ for all $k$ in $\mathbb{H}_{p}^{1}$, Jensen's inequality and a standard argument of lower semi-continuity achieve the proof of the following convergence result which supplies our asymptotic models in the form of variational problems $\left(\bar{P}_{p}\right)$ :

Theorem 2.1. When $\varepsilon$ tends to zero, $\left(u_{p}^{\varepsilon},\left(k\left(\varepsilon, \varphi_{p}^{\varepsilon}\right)\right)^{1}\right)$ converges strongly in $H^{1}\left(\Omega ; \mathbb{R}^{3}\right) \times L^{2}\left(\mathbb{S}^{2} \times \mathbb{R}^{3-p_{1}}\right)$ toward $\left(\bar{u}_{p},\left(e\left(\widehat{\bar{u}_{p}}\right), \bar{E}_{p}\right)\right)$ where $\left(\bar{u}_{p}, \bar{E}_{p}\right)$ is the unique solution to:

$$
\left(\bar{P}_{p}\right) \quad\left\{\begin{array}{l}
\text { Find }(u, E) \in\left(p_{1}-1\right)\left(p_{2}-1\right)\left(0, \varphi_{0}\right)+\bar{V} \times \mathcal{E}_{p} \quad \text { such that } \\
\overline{\mathcal{M}}_{p}\left((u, E),\left(v, E^{\prime}\right)\right)=\overline{\mathcal{L}}_{p}\left(v, E^{\prime}\right) \quad \forall\left(v, E^{\prime}\right) \in \bar{V} \times \mathcal{E}_{p}
\end{array}\right.
$$

where

$$
\begin{aligned}
& \mathcal{E}_{(1,1)}:=\left\{E \in L^{2}\left(S ; \mathbb{R}^{2}\right) ; \exists ! \phi:=(\nabla)^{-1} E \in H_{m}^{1}(S):=\left\{\psi \in H^{1}(S) ; \int_{S} \psi(\hat{x}) \mathrm{d} \hat{x}=0\right\} ; E=\nabla \phi\right\} \\
& \mathcal{E}_{(1,2)}=\{0\}, \quad \mathcal{E}_{(2,1)}=L^{2}(S), \quad \mathcal{E}_{(2,2)}=\{0\} \\
& \overline{\mathcal{M}}_{p}\left((u, E),\left(v, E^{\prime}\right)\right):=\int_{\Omega} a e(u) \cdot e(v) \mathrm{d} x+\int_{S} \widetilde{\mathcal{M}}_{p}(e(\hat{u}), E) \cdot\left(e(\hat{v}), E^{\prime}\right) \mathrm{d} \hat{x} \\
& \overline{\mathcal{L}}_{p}\left(v, E^{\prime}\right)=\int_{\Omega} f \cdot v \mathrm{~d} x+\int_{\Gamma_{1}} F \cdot v \mathrm{~d} H_{2}+\int_{S} G \cdot v \mathrm{~d} \hat{x}+\left(2-p_{1}\right) \int_{S} q(\nabla)^{-1} E^{\prime} \mathrm{d} \hat{x}
\end{aligned}
$$

and any element of $\mathbb{H}_{p}^{1}$ is understood as an element of $\mathbb{S}^{2} \times \mathbb{R}^{3-p_{1}}$.

When $p_{2}=1$, the model involves an additional state variable to the sole displacement, which is the limit of the average in the transverse direction of the efficient components of the electrical field in the patch. As it can be eliminated, $\left(\bar{P}_{p}\right)$ models the equilibrium of the genuine body subjected to the loading $(f, F, G)$ and reinforced along $S$, this reinforcement being nonlocal if $p=(1,1)$.

\section{Concluding remarks}

When $p=(1,2)$ or $p=(2,1)$, the electric data $q$ or $\varphi_{0}$ do not have any influence on the limit model which corresponds to a purely elastic surface reinforcement of the body along $S$. However, the characteristics of this reinforcement may depend on the dielectric or piezoelectric coefficients as much as such terms appear in the expression of $\mathcal{M}_{p}$ (see [1], where the systematic influence of crystal symmetries has been carried out). On the contrary, electrical data $q$ or $\varphi_{0}$ plays a role in models $(1,1)$ and $(2,2)$. More precisely, $f, F$ and $G$ being fixed, the mapping $\varphi_{0} \mapsto S_{(2,2)} \varphi_{0}=\bar{u}_{(2,2)}$ is one-to-one, it is "theoretically" possible to determine what could be the electrical potential to apply on $S^{\varepsilon}$ in order to get a desired displacement in the range of $S_{(2,2)}$. An approximate procedure may be done easily by finite elements. Another application is that the patch may shift the spectrum of the body in an interesting way, that is why we may regard the patch as an 
actuator. When $p=(1,1)$ the mapping $q \mapsto \mathrm{S}_{(1,1)} q=\bar{u}_{(1,1)}$ is also one to one, thus the measurement of $q^{\varepsilon}$ may supply the knowledge of the state of displacements: the patch acts as a sensor!

By using the same technique of averaging the strain and electrical fields in the transverse direction it is possible to treat the easier case, from the mathematical point of view, of piezoelectric patches embedded inside an elastic body.

\section{References}

[1] T. Weller, C. Licht, Asymptotic modeling of thin piezoelectric plates, Ann. Solid Struct. Mech 1 (2010) 173-188.

[2] J.N. Reddy, On laminated composite plates with integrated sensors and actuators, Eng. Struct. 21 (1999) 568-

593. 